and discrimination, which in turn affect their access to housing, employment, healthcare and put them in a high risk of STD. These characteristics are shared with sex workers, injecting drug users and men who have sex with men. However, gender identity has led to a particular combination of vulnerabilities, ultimately resulting in a much higher risk of HIV infection among the male-to-female transgender population as mentioned above.

Methods We conducted an exploratory descriptive study and collected data with surveys, in-depth interviews, focus groups and ethnographic strategies with 18 transgender women. The collected information was analysed, taking into account the following dimensions of interpretation: 1-Love, erotic, and sexual relations. 2- Gender and sexual identities. 3-Sexual behaviour and practices. 4-Self-care, HIV-risk perception and life goals.

Results Transgender women believed that they needed to perform an overtly feminine identity (hyperfeminine). They think this performance involves taking sexual risks assigned traditionally by the social space such as street prostitution. In this role transgender women's negotiation power is significantly reduced. Many transgender women assumed and accepted this gender disparity social structure that increased their vulnerability to STI/HIV.

Conclusions This research intended to identify the most significant ways in which sexuality is represented and imagined within the culture of transgender women in Colombia. Special emphasis was placed on discovering transgender women's logics, or thinking processes, which are capable of perpetuating their high vulnerability to HIV/AIDS. Transgender women in Colombia construct their gender identities within contexts of stigmatisation, social marginalisation, and multiple symbolic and physical forms of violence. Our work critiques approaches based on androcentrism that perceive gender identities as essential and fixed, and assume sexual binaries that oppress the lived experience of human bodies. We propose a peer-led educational strategy focused on the deconstruction and reconstruction of gender identities and interventions with transgender women that reduce stigma through enhancing autonomy, joy, and self-care. We argue that these are the central elements for a "life with quality" in which equal citizenship can be exercised.

\section{P2-S5.04 BEHAVIOURAL RISKS AND HIV/AIDS KNOWLEDGE IN ADOLESCENT STREET CHILDREN AND THEIR HEALTH IMPLICATIONS}

doi:10.1136/sextrans-2011-050108.346

${ }^{1} \mathrm{~F}$ Naseem, ${ }^{2} \mathrm{R}$ Ahmad, ${ }^{3} \mathrm{~A}$ Rashid. ${ }^{1}$ Degree College for Women, Rawalpindi, Pakistan; ${ }^{2}$ Street NGO, Quetta, Pakistan; ${ }^{3}$ PMAS Arid Agriculture University, Rawalpindi, Pakistan

Background In Pakistan social and ethical bindings are major constraints to launch effective awareness campaign or vaccination trial against HIV transmission. Lack of awareness coupled with high risk behaviour could result in AIDS epidemic among adolescents and street children as in these groups coercive and transactional sex is common. We aimed to examine adolescent's behavioural risks and their knowledge about HIV/AIDS. Sources of information regarding HIV/ AIDS were analysed for risk behaviour in relation to social factors. Methods Cross-sectional study was conducted in rural and urban areas of Rawalpindi to gather information from street children ( $n=148$, mean age 17 years). Multivariate analysis was performed to see effect of residential site and gender on AIDS concepts.

Results Among surveyed population, 14\% had heard of HIV vaccine and $50 \%$ believe HIV vaccine could develop infection. About 33\% ever had sex and $10 \%$ revealed $>1$ partner per year. $5 \%$ had knowledge about HIV status of partner see Abstract P2-S5.04 Table 1. A high degree of risky behaviour activities correlated with those
Abstract P2-S5.04 Table 1 Multivariate ANOVA for HIV/AIDS knowledge

\begin{tabular}{lcccc}
\hline & \multicolumn{2}{c}{ Main effect } & & $\begin{array}{l}\text { Interaction } \\
\text { Site } \times \text { Gender }\end{array}$ \\
\cline { 2 - 3 } & Site & Gender & & S \\
\hline MANOVA test (Pillais F value) & 2.17 & 4.45 & 1.43 \\
HIV/AIDS transmission route & $<1$ & $<1$ & $<1$ \\
Discussion about HIV/AIDS & 1.2 & 2.21 & 1.53 \\
Behavior risks & $<1$ & $<1$ & 2.25 \\
\hline
\end{tabular}

children who lived in rural areas. Urban area street children were found to explore more sources to grasp HIV information. We found limited availability of documented information to compare the present observations.

Conclusions Both the adolescents and street children with poor knowledge about HIV are involved in risky behaviour. Our study propelled the need to consolidate, where relevant, community mobilisation for HIV prevention research throughout the country. Moreover, cross-country documentation must be improved for wider sharing of knowledge. A planned approach with educational sessions seems imperative for developing an effective sexual health safety program.

\section{P2-S5.05 RISK PROFILES OF WINNIPEG STREET POPULATIONS: A LATENT CLASS ANALYSIS}

doi:10.1136/sextrans-2011-050108.347

${ }^{1} \mathrm{~S}$ Hopfer, ${ }^{2} \mathrm{~J}$ Wylie, ${ }^{1} \mathrm{X}$ Tan. ${ }^{1}$ Pennsylvania State University, State College, USA; ${ }^{2}$ Cadham Provincial Laboratory, University of Manitoba, Winnipeg, Canada

Background Sexually transmitted and bloodborne infection (STBBI) risk is multifaceted and can involve a complex interplay between sexual behaviours, substance abuse and mental health conditions. In Winnipeg, Manitoba Canada we conducted a study to better understand the interconnectedness and overlap of these conditions and behaviours.

Methods Data from the Social Network study phase III (SNS III) were collected in the fall and winter of 2009 using semi-structured in-person interviews $(n=600)$. Sampling was by respondent driven sampling and targeted street populations. The average mean age was $37(\mathrm{SD}=14.8)$ and the gender distribution was relatively equal (males constituted 53\%). Latent class analysis was used to identify unobserved or latent subgroups (ie, risk profiles) to explore the extent of overlap between risky sexual behaviours, substance use choice (crack, alcohol, solvents, injection drug use), and mental health conditions. Six individual level items constituting risky behaviours and five network or environmental level risk behaviours were used in the latent class analysis. Individual items included: Ever diagnosed with a mental health condition, ever used crack, daily binge drinking, ever used solvents, ever injected drugs and knowing your sex partner has multiple other sex partners while social network items included: the proportion of your social network members who drink alcohol, use crack, sniff solvents, inject drugs, or are sex partners. Fit indices of G2, AIC, and BIC were used in assessing model fit. Additionally, the model fit was assessed by examining the relationship between items and their conditional latent class by strength of homogeneity (closeness to 0 or 1 ) and by whether there was evidence of good separation of latent classes.

Results The 2-, 3-, 4-, and 5-class LCA models were compared. Goodness of fit indices favoured the 4-class model. For the 4-class model indices were: $\mathrm{G} 2=1115, \mathrm{df}=2000, \mathrm{AIC}=1209, \mathrm{BIC}=1415$. Class prevalence of the 4 latent classes were: $31 \%$ were at high risk for all individual and network items, 25\% constituted another latent class labelled as low-risk, $21 \%$ constituted a subgroup who were labelled as "loners" and exhibited high risk for mental 